\title{
Energy spread tuning of a laser-plasma accelerated electron beam in a magnetic chicane
}

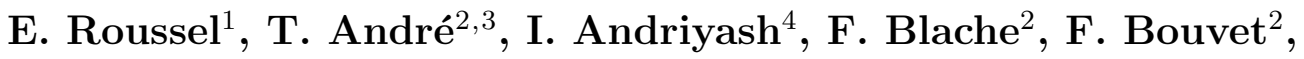 \\ S. Corde ${ }^{5}$, D. Oumbarek Espinos ${ }^{2,3,6}$, A. Ghaith ${ }^{2,3}$, J.-P. \\ Goddet $^{5}$, C. Kitegi ${ }^{2}$, O. Kononenko ${ }^{5}$, M. Labat ${ }^{2}$, G. Lambert ${ }^{5}$, \\ A. Lestrade ${ }^{2}$, A. Loulergue ${ }^{2}$, F. Marteau ${ }^{2}$, O. Marcouillé ${ }^{2}$, M.

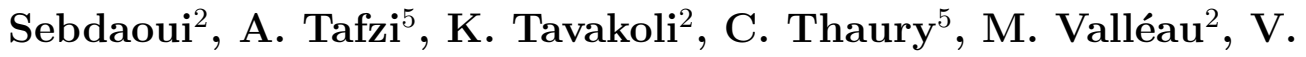 \\ Malka $^{4,5}$ and M.-E. Couprie ${ }^{2}$ \\ ${ }^{1}$ Univ. Lille, CNRS, UMR 8523 - PhLAM - Physique des Lasers, Atomes et \\ Molécules, F-5900 Lille, France \\ ${ }^{2}$ Synchrotron SOLEIL, Saint-Aubin, Gif-sur-Yvette, F-91192, France \\ ${ }^{3}$ Université Paris-Saclay, F-91190 Paris, France \\ ${ }^{4}$ Department of Physics of Complex Systems, Weizmann Institute of Science, \\ Rehovot 761001, Israel \\ ${ }^{5}$ LOA, ENSTA Paris, CNRS, Ecole Polytechnique, Institut Polytechnique de Paris, \\ 828 Boulevard des Maréchaux, 91762 Palaiseau, France, Université Paris-Saclay, \\ F-91190 Paris, France \\ ${ }^{6}$ Graduate School of Engineering, Osaka University, 2-8 Yamadaoka, Suita, Osaka \\ 565-0871, Japan \\ E-mail: eleonore.roussel@univ-lille.fr
}

14 March 2020

\begin{abstract}
Laser-plasma accelerators (LPA) deliver relativistic electron beams with high peak current and low emittance, with energies up to the GeV-level in only few centimetres. However, the divergence and the energy spread of these beams remain too large for potential light source applications. A magnetic transfer line can be used to manipulate the electron beam phase-space and select the transmitted energies with a slit located at its center. We will show that with a proper focusing optics along the line, one can tune the energy spread of the beam at a given energy without loss and produce undulator radiation with controlled bandwidth. We present analytic studies, numerical simulations and experimental results on the controled electron beam transport and application to undulator radiation with a control of the bandwidth.
\end{abstract}

\section{Introduction}

Laser-Plasma Acceleration (LPA) is a promising technology as an alternative for miniaturising the conventional radio-frequency-based accelerators. In recent LPA schemes, a high-power femtosecond laser is focused into a gas target and excites a 
plasma-density wave with ultra-high accelerating fields much larger than in the radiofrequency cavities of conventional accelerators [1]. In such compact accelerators, trapped electrons from the ambient plasma can be accelerated up to relativistic energies within a millimetre distance [2]. Progress in LPAs have made possible the generation of quasimono energetic electron beams with ultra relativistic energies up to multi-GeV $[3,4,5]$. However, the characteristics of the produced electron beams strongly depend on how electrons are injected into the accelerating plasma structures. In the past decade, different strategies of controlled injection schemes have been proposed such as density transition injection $[6,7,8,9,10]$, multipulse colliding injection $[11,12]$, ionisation injection $[13,14,15]$ and plasma lens enhancement of self-injection $[16,17,18,19]$ but the current lowest relative energy spread reported is still at the percent level [20].

The independent control over electron beam parameters such as energy spread, charge, emittance and divergence is still a key challenge towards potential LPA applications such as x-ray free-electron lasers (FELs) [21]. The intrinsic large divergence (few milliradians) and energy spread (few percent) of the electron beam [6, 22] dramatically limit the capacity for an LPA beam to sustain FEL gain. Several techniques, either focused on the LPA development or based on conventional accelerator strategies, can be implemented to reach the mandatory Pierce parameter $\rho_{F E L}$ [23] qualifying the FEL gain: use plasma lenses [24, 25, 26, 27] and/or high gradient quadrupoles in the immediate vicinity of the LPA source to handle the large divergence [28, 29], use a chicane [30, 31] or a transverse gradient undulator [32, 33] to lower the slice energy spread, and/or use a chromatic matching [34] to synchronize the beam maximum density with the radiation pulse propagation inside the undulator.

In view of approaching FEL application, experimental demonstration with existing LPA performance are required for a better understanding of LPA-based FEL requirements. For that purpose, the COXINEL line [30] has been designed to operate an LPA in combination with a conventional transfer line based on multiple magnetic devices. This permits to facilitate and handle the transport of such non trivial beams and qualify them for FEL application.

In this paper, we present a method inspired from conventional accelerators where the energy spread is handled in a magnetic transfer line using a slit inserted between

the second and third dipole magnet of a chicane. As a main result, the control of the energy spread permits to improve the spectral purity of the spontaneous undulator radiation. Experimental results on electron beam transport and undulator radiation on the COXINEL line are confronted with numerical simulations based on experimental LPA electron beams observations.

\section{Laser-plasma accelerated electrons at the gas jet exit}

The 60 TW, 30 fs Titanium:Sapphire laser from Salle Jaune at Laboratoire d'Optique Appliquée (LOA) is focused into a supersonic gas jet of $\mathrm{He}$ and $\mathrm{N}_{2}$ mixture. The ionisation injection regime [35] is considered because of its simplicity and robustness 
over daily operation. The electron beam properties are characterised using an electron spectrometer composed of a dipole magnet and a scintillator screen for energy distribution measurements. An estimation of the charge density per energy can be obtained from the calibration of the number of photons created per incident electron on the scintillator screen [36].

Figure 1a shows a series of measured single-shot energy distributions. Electron beams with a broadband energy distribution ranging from few tens up to $200 \mathrm{MeV}$ are generated with a total average charge of $75.5 \mathrm{pC}$ (Fig. 1b). While the horizontal distribution permits to reconstruct the energy distribution, the slice divergence of the beam can be deduced from the local distribution on the vertical axis. The vertical slices are fitted with a Gaussian distribution. The slice vertical divergence distribution is shown in Fig. 1c.

Even though the LPA provides electron beams with a large energy spread, only a small part of the electron beam with the adequate energy is transported along the beamline. Moreover, for lightsources applications with quasi monochromatic light, such as undulator radiation or FEL, the proper quantity is not the total charge of the electron beam but the charge density contributing to the radiation process, in a given spectral bandwidth, that is equal to $1 / N_{U}$ for spontaneous undulator radiation with $N_{U}$ the number of undulator period. In that case, the relevant quantities are the charge per energy slice and the slice divergence at the nominal energy, which is, in our case, equal to $176 \mathrm{MeV}$ (see next section). Figures 1d,e show the evolution of the charge, approximately equal to $0.2 \mathrm{pC} / \mathrm{MeV}$, and the vertical divergence, around $1.6 \mathrm{mrad}$ ( $\mathrm{rms}$ ) within a $1 \mathrm{MeV}$ slice around the nominal energy. The horizontal divergence is deduced from the vertical one by applying a coefficient proportional to the ratio of the beam size $\left(r=\sigma_{x} / \sigma_{z}\right)$ measured at the first scintillator screen after a direct drift from the gas jet. The aspect ratio between the horizontal and vertical size is, on average, equal to 1.86. This asymmetric beam distribution may come from the horizontal polarisation of the laser [37]. The shot-to-shot pointing fluctuations are of the order of $3.5 \mathrm{mrad}$ (rms).

\section{COXINEL: a magnetic transfer line for laser-plasma accelerated beams}

The COXINEL project $[38,39,40,41]$ aims at qualifying the LPA electron beams for FEL applications using a dedicated manipulation line. The beamline is depicted in Fig. 2a. The magnetic transfer line has been initially designed for a $1 \%$ energy spread and a 1 mrad divergence LPA beam and built at Synchrotron SOLEIL. The line is currently installed in Salle Jaune (LOA) and has been upgraded to handle the performance of the available LPA electron beams. A triplet of permanent quadrupole magnets with adjustable strength $[42,29,43]$, located at $5 \mathrm{~cm}$ from the LPA gas jet, is used to strongly focus the electron beam in order to mitigate the divergence. A magnetic chicane, also called demixing chicane, is then used to stretch the electron bunch in order to longitudinally sort the energy along the beam at the cost of a decrease of the peak current $[31,30,34]$. Finally, a quadruplet of electromagnetic quadrupoles focuses the 

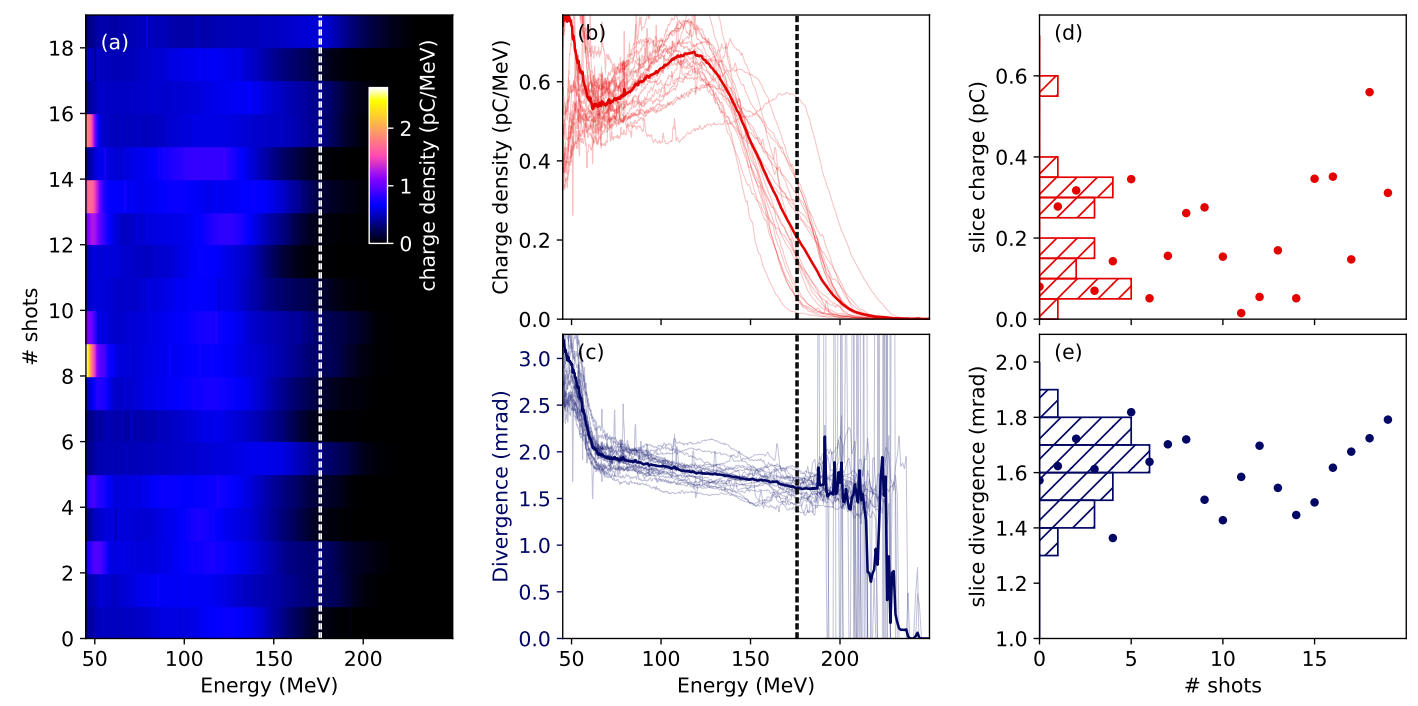

Figure 1. Experimental laser-Plasma accelerated electrons properties. (a) Series of 20 single-shot energy distributions. (b) Average (thick line) and single-shots (light lines) charge density per energy. (c) Average (thick line) and single-shots (light lines) divergence (rms) distribution per energy. Charge (d) and divergence (rms) (e) within a $1 \mathrm{MeV}$ slice around the $176-\mathrm{MeV}$ reference energy (vertical dashed lines in a-c). The histograms show the charge and divergence distributions.

beam at the undulator center.

The beam transport is based on a source-to-image optics in which the focusing magnification depends on the energy range (Figs. 2b,c). In the previous work [44], the demixing chicane was combined with a specific chromatic matching to synchronise the beam maximum density with the radiation pulse propagation along the undulator [34]. In the present configuration, the electron beam is focused in the horizontal plane at the center of the chicane (Fig. 2e) and then refocused in both horizontal and vertical planes, at the undulator center (Figs. 2b,c). Thanks to the horizontal dispersion at the chicane center, the adjustable-aperture slit permits to energy select the transmitted electrons along the beamline. The effect of the slit will be described in the following. The numerical simulation is done using particle tracking with initial conditions deduced from the experimental observations of the LPA beam (Fig. 1).

The LPA electron beam has been successfully transported along the COXINEL line (Figs. 2h-k). In order to mitigate pointing and alignment errors, a beam pointing alignment compensation method (BPAC) has been developed on purpose [44]. At the slit entrance, the shot-to-shot fluctuations in the horizontal plane are of the order of $167 \mu \mathrm{m}$ (Fig. 2i). 


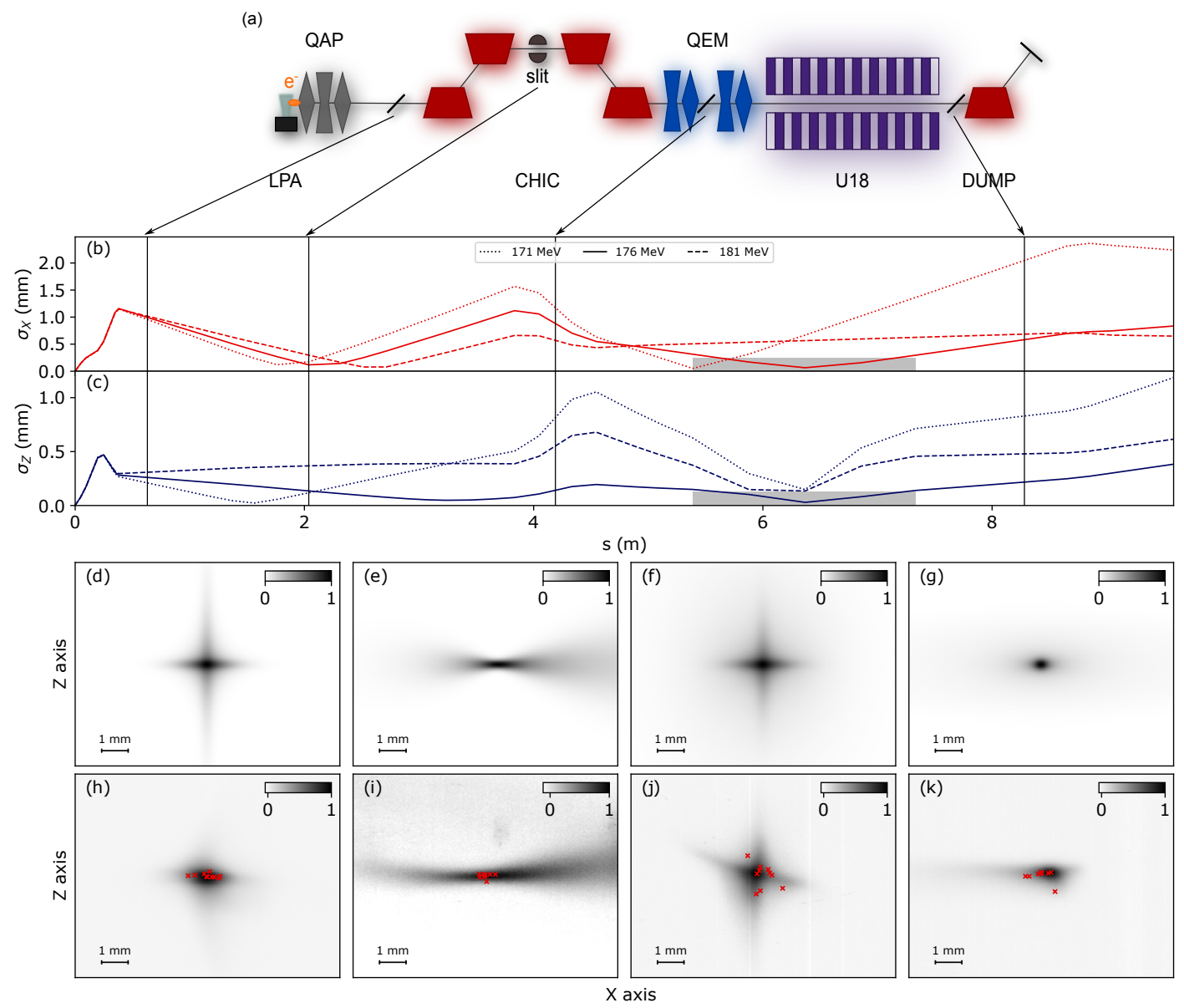

Figure 2. (a) COXINEL experimental setup. Electrons generated in a gas mixture jet (LPA) pass through a triplet of adjustable permanent quadrupole magnets (QAP) and then in a magnetic chicane (CHIC) where a slit permits electron energy selection. They are then focused using a quadruplet of quadrupoles (QEM) in an in-vacuum undulator (U18). At the end of the undulator, they are dumped by means of a bending magnet (DUMP). Transverse electron beam distributions can be imaged on scintillator screens (black thick lines). Horizontal (b) and vertical (c) beam enveloppe for 171 (dot), 176 (solid) and 181 (dashed) MeV. Transverse beam distributions along the beamline: numerical simulations ( $\mathrm{d}-\mathrm{g}$ ) and experimental observations $(\mathrm{h}-\mathrm{k})$. Numerical simulations are done using electron beam parameters from Tab. 1 and average energy and divergence distributions from Fig. 1. The red crosses represent the shot-to-shot pointing fluctuations at the scintillator screens.

\section{Electron beam monochromator}

Due to its broadband energy distribution, the LPA beam is not usable as it is for light source applications that require low energy spread such as FEL. In parallel to the improvements of high-quality LPA electron beams generation, a proper manipulation of the phase-space of the LPA beams along the beamline can help in reducing the energy spread to approach the properties of conventional accelerators. For that purpose, we use 
an electron beam monochromator composed of a slit, i.e. collimator, with adjustable aperture located between the second and the third dipole magnet of the demixing chicane (Fig. 2a) [45].

The effect of the slit aperture is studied using particle tracking simulations [46]. The magnetic line is considered ideal. A deeper study of the influence of more realistic magnets on the emittance growth of the beam is presented in [47], and sensitivity studies to beam fluctuations can be found in $[48,49]$. The influence of collective effects on the slice emittance has also been examined in [50], showing that the decompression of the bunch significantly reduces the influence of the space charge on the slice emittance during the transport. The experimental LPA energy and divergence distributions (Figs. 1b,c) combined with the electron parameters summarised in Tab. 1 are used to generate the initial electron distributions as input of the numerical simulations. Since the real 6D phase-space distribution is unknown and not accessible experimentally, the simulations are done assuming independent Gaussian transverse distributions.

\begin{tabular}{rl}
\hline Nominal energy $E_{0}$ & $176 \mathrm{MeV}$ \\
Normalized emittance $\varepsilon_{n x, n z}$ & $1 \mathrm{~mm} . \mathrm{mrad}$ \\
Bunch length $\Delta T_{e}(\mathrm{FWHM})$ & $30 \mathrm{fs}$ \\
Total charge $Q_{t o t}$ & $75.5 \mathrm{pC}$ \\
Charge density $\left(@ E_{0}\right) d Q / d E$ & $0.2 \mathrm{pC} / \mathrm{MeV}$ \\
Vertical divergence $\left(@ E_{0}\right) \sigma_{z_{0}^{\prime}}$ & $1.6 \mathrm{mrad}(\mathrm{rms})$ \\
Ratio $r=\sigma_{x} / \sigma_{z}$ & 1.86 \\
Undulator period $\lambda_{U}$ & $18.16 \mathrm{~mm}$ \\
Undulator parameter $K$ & 1.88 \\
Numbrer of undulator period $N_{U}$ & 107 \\
\hline
\end{tabular}

Table 1. COXINEL line, electron beam and numerical simulations parameters.

At the chicane center, the electron beam is horizontally dispersed, i.e. the horizontal position of the electrons is correlated with the electron energy, and the nominal energy of $176 \mathrm{MeV}$ is focused in both horizontal and vertical planes (Fig. 3a). At the first order, the correlation between the horizontal position $x$ and the relative beam energy deviation $\delta$ is given by:

$$
x(\delta)=x_{0}+R_{16}^{\text {slit }} \delta
$$

where $R_{16}^{\text {slit }}$ is the transport matrix coefficient [51] at the slit position, also called dispersion function $D_{x}$, and $x_{0}$ is the horizontal displacement of the nominal energy. The insertion of a slit, centred around the nominal energy position, permits then to transversally select the transmitted energies. Thanks to the horizontal focusing (Fig. 2b), the nominal energy is transmitted without loss (Fig. 3b). According to Eq. (1), the transmitted relative energy bandwidth $\Delta \delta$ is given by:

$$
\Delta \delta=\frac{\Delta x_{\text {slit }}}{\left|R_{16}^{\text {slit }}\right|}
$$


with $\Delta x_{\text {slit }}$ the aperture of the slit. The evolutions with the slit aperture of the total transmitted charge and energy spread of the beam at the undulator center are shown in Figs. 3c,d. As the slit aperture is closed, the total transmitted charge and energy spread decrease linearly.
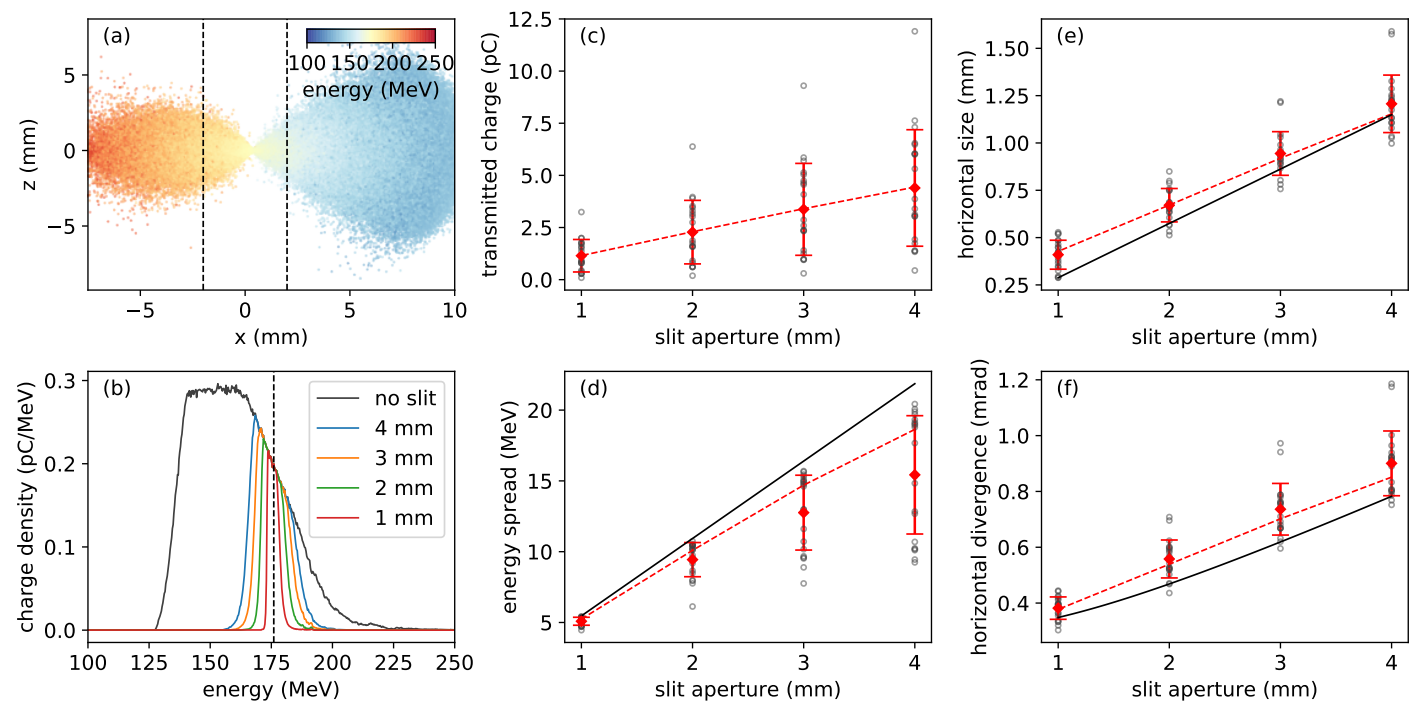

Figure 3. Impact of the electron beam monochromator. (a) Transverse-energy distribution of the electron bunch in presence of horizontal dispersion at the center of the chicane. The vertical dashed lines represent the borders of the maximum slit aperture (4 mm full width). (b) Energy distributions versus slit aperture. The vertical dashed line highlights the $176 \mathrm{MeV}$ reference energy. (c) Total charge at the center of the undulator versus slit aperture. (d) Evolution of the full width at half maximum (fwhm) energy spread versus slit aperture. Evolution of the rms horizontal beam size (e) and rms horizontal divergence (f) at the undulator center versus slit aperture. Numerical simulations from single-shot distributions (grey dots) and average of the single-shot results (red). Numerical simulations from average distribution (red dashed line) and analytical formula (black solid line). The transport matrix coefficients are: $R_{16}^{\text {slit }}=-32.2 \mathrm{~mm}$, and at the undulator center: $R_{11}=9.6, R_{12}=-5.3 \cdot 10^{-3} \mathrm{~m}$, $R_{116}=-15.6, R_{126}=-7.3 \mathrm{~m}, R_{21}=6.3 \mathrm{~m}^{-1}, R_{22}=0.1, R_{216}=4.5 \cdot 10^{-2} \mathrm{~m}^{-1}$, $R_{226}=-4.6$.

Up to second order in dispersion free section, a focusing transport in the horizontal plane is given by:

$$
\left(\begin{array}{c}
x \\
x^{\prime}
\end{array}\right)=\left[\left(\begin{array}{ll}
R_{11} & R_{12} \\
R_{21} & R_{22}
\end{array}\right)+\delta\left(\begin{array}{ll}
R_{116} & R_{126} \\
R_{216} & R_{226}
\end{array}\right)\right] \cdot\left(\begin{array}{c}
x_{0} \\
x_{0}^{\prime}
\end{array}\right)
$$

using the standard transport matrix notation [51]. The first matrix $\left(R_{i j}\right)$ corresponds to the linear part and the second matrix $\left(R_{i j 6}\right)$ takes into account the chromatic second order perturbation. $\left(x_{0}, x_{0}^{\prime}\right),\left(x, x^{\prime}\right)$ are respectively the initial and final horizontal coordinates (position-angle). Assuming an initial Gaussian distribution without any correlation, the rms beam size and divergence at the focal point can be approximated 
Energy spread tuning of a laser-plasma accelerated electron beam in a magnetic chicane8

by:

$$
\begin{aligned}
\sigma_{x} & \approx \sqrt{R_{11}^{2} \sigma_{x 0}^{2}+R_{12}^{2} \sigma_{x^{\prime} 0}^{2}+R_{116}^{2} \sigma_{x 0}^{2} \sigma_{\delta}^{2}+R_{126}^{2} \sigma_{x^{\prime} 0}^{2} \sigma_{\delta}^{2}} \\
\sigma_{x^{\prime}} & \approx \sqrt{R_{21}^{2} \sigma_{x 0}^{2}+R_{22}^{2} \sigma_{x^{\prime} 0}^{2}+R_{216}^{2} \sigma_{x 0}^{2} \sigma_{\delta}^{2}+R_{226}^{2} \sigma_{x^{\prime} 0}^{2} \sigma_{\delta}^{2}}
\end{aligned}
$$

Thanks to the slit, the reduction of the energy spread leads to a reduction of the horizontal beam size and divergence at the center of the undulator by limiting the chromatic effects during the transport (Figs. 3e,f).

\section{Undulator radiation}

When targeting light source applications, a rather monochromatic emission is usually expected. The control of the energy spread can have a strong impact on the spectral properties of spontaneous undulator radation. The radiation emitted by electrons wiggling in a periodic magnetic field constructively interfere at the fundamental resonance wavelength (and its harmonics):

$$
\lambda_{R}=\frac{\lambda_{U}}{2 \gamma_{0}^{2}}\left[1+\frac{K_{U}^{2}}{2}+\left(\gamma_{0} \theta\right)^{2}\right]
$$

where $\lambda_{U}$ is the undulator period, $K_{U}$ the deflection parameter proportional to the magnetic field and the undulator period, $\gamma_{0}$ is the Lorentz factor and $\theta$ the observation angle. The undulator radiation presents a characteristic angulo-spectral parabolalike distribution with a high spectral purity and brilliance assuming negligible multielectron contributions $[52,53]$. Indeed, the spectral broadening due to multi-electron contributions are presented in the following [54].

\subsection{Relative bandwidth}

The natural on-axis bandwidth of an undulator is inversely proportional to the number of undulator periods $N_{U}$ :

$$
\left.\frac{\Delta \lambda}{\lambda}\right|_{0}=\frac{1}{N_{U}} .
$$

However, an inhomogeneous broadening of the undulator radiation bandwidth can result from multi-electron contributions coming from the electron beam parameters such as the large energy spread or horizontal divergence of the beam. The relative energy spread of the beam symmetrically broadens the bandwidth as follows:

$$
\left.\frac{\Delta \lambda}{\lambda}\right|_{\sigma_{\gamma}} \simeq 2 \times \frac{\sigma_{\gamma}}{\gamma_{0}} .
$$

In addition, the divergence brings a non-negligible contribution to the broadening of the bandwidth on the long-wavelength side and can be estimated by:

$$
\left.\frac{\Delta \lambda}{\lambda}\right|_{\sigma_{x^{\prime}}} \simeq \frac{\left(\gamma_{0} \sigma_{x^{\prime}}\right)^{2}}{1+\frac{K_{U}^{2}}{2}}
$$


Assuming independent multi-electron contributions, the on-axis undulator radiation bandwidth can be obtained from the quadratic sum of the previous contributions (Eqs. $(7,8,9))[54,55]$ :

$$
\frac{\Delta \lambda}{\lambda}=\frac{1}{2} \sqrt{\left.\frac{\Delta \lambda}{\lambda}\right|_{0} ^{2}+\left.\frac{\Delta \lambda}{\lambda}\right|_{\sigma_{\gamma}} ^{2}}+\sqrt{\left.\frac{1}{4} \cdot \frac{\Delta \lambda}{\lambda}\right|_{0} ^{2}+\left.\frac{1}{4} \cdot \frac{\Delta \lambda}{\lambda}\right|_{\sigma_{\gamma}} ^{2}+\left.\frac{\Delta \lambda}{\lambda}\right|_{\sigma_{x^{\prime}}} ^{2} .}
$$

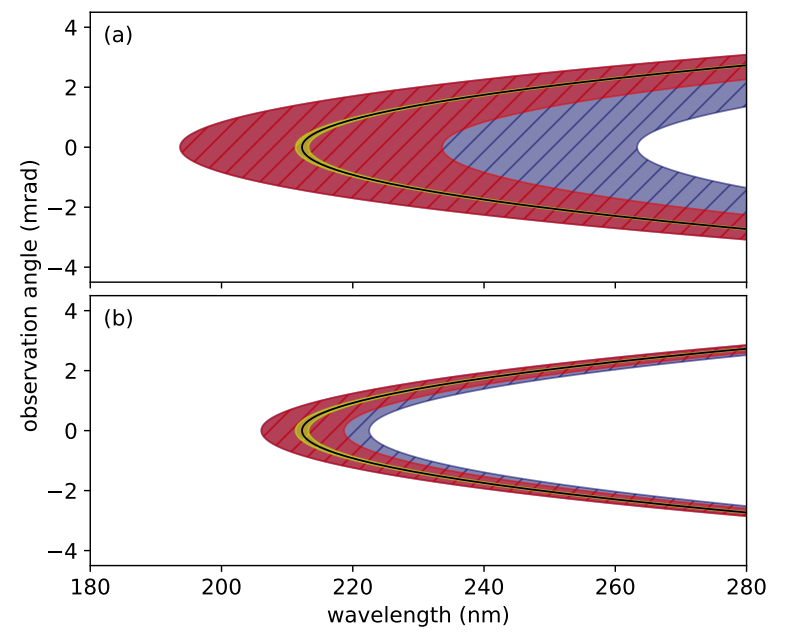

Figure 4. Resonance undulator radiation wavelength (black line), homogeneous bandwidth (yellow area), energy spread broadening contribution (red area) plus horizontal divergence contribution (blue area), for a 4-mm (a) and a 1-mm (b) slit aperture. The undulator parameters are: $\lambda_{U}=18.16 \mathrm{~mm}$ and $K_{U}=1.88$.

Figure 4 illustrates the different broadening contributions to the undulator radiation bandwidth for two extreme slit apertures: the maximum aperture of $4 \mathrm{~mm}$ (Fig. 4a) and the minimum aperture of $1 \mathrm{~mm}$ (Fig. 4b). In absence of the electron monochromator, the transmitted energy spread is so large (around $17.5 \mathrm{MeV}$ rms, Fig. 3b) that the on-axis undulator radiation will span over a window close to $100-n m$ width.

As the slit is closed, the electron beam energy spread, size and divergence are reduced at the undulator center (Figs. 3, Eqs. (4, 5)). According to Eq. (10), the expected radiation bandwidth decreases (Fig. 5a). This decrease is dominated by the energy spread contribution (Fig. 5a, red). At larger slit width, the beam divergence contributes to an additional broadening of the bandwidth (Fig. 5a, blue).

\subsection{Spectral peak brilliance}

The spectral peak brilliance $B_{p k}$ is a typical figure of merit for accelerator-based light sources. It describes the intensity of the light source taking into account its spectral purity and opening angle. For radiation sources with only partial coherence such as spontaneous undulator emission, the spectral peak brilliance can be expressed as [56]:

$$
B_{p k} \propto \frac{N_{e}}{4 \pi^{2} \sigma_{x} \sigma_{x^{\prime}} \sigma_{z} \sigma_{z^{\prime}} \Delta t(\Delta \lambda / \lambda)},
$$


Energy spread tuning of a laser-plasma accelerated electron beam in a magnetic chicane10
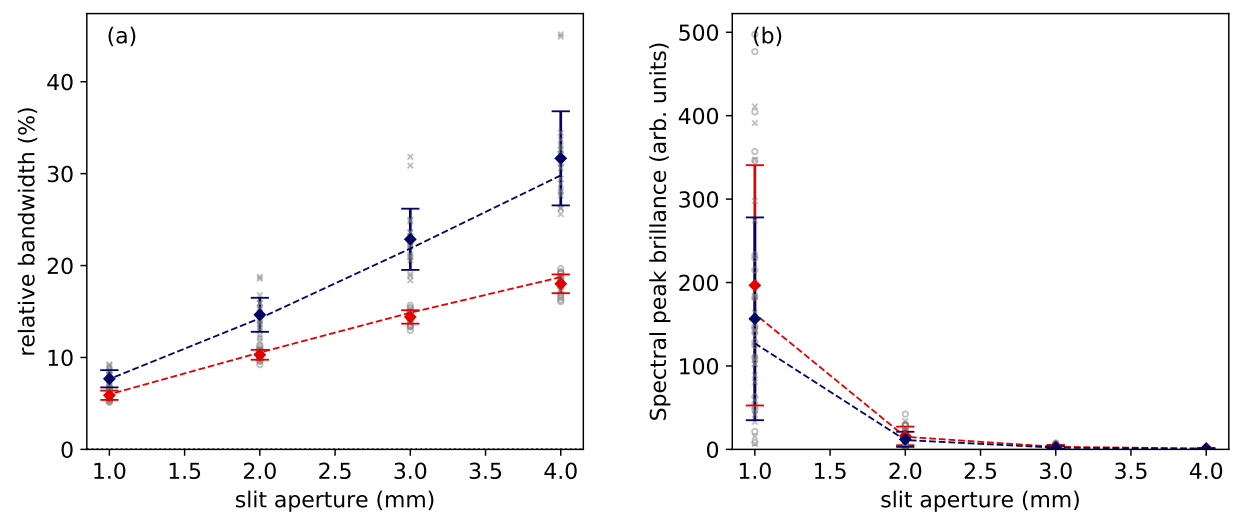

Figure 5. Theoretical undulator radiation relative bandwidth (a) and spectral peak brilliance (b) versus electron slit aperture. (Homogeneous linewidth (a-black dot line), relative energy spread inhomogeneous broadening (red) plus horizontal divergence contribution (blue)).

where the number of photons is proportional to the number of electrons $N_{e}$ in the bunch of duration $\Delta t, \sigma_{x, z, x^{\prime}, z^{\prime}}$ are the transverse rms sizes and angular divergences of the source.

Figure 5b shows the relative improvement of the spectral peak brilliance versus slit aperture, taking into account all the electron beam parameters in Fig. 3. As the slit is closed, the transmitted beam charge is reduced but the charge at the energy of interest is maintained. The relative bandwidth is also reduced as the slit limits the transmitted energy spread. Simultaneously, the electron beam sizes and divergences at the undulator center are also reduced thanks to the energy spread control. All these combined effects lead to an enhancement of the spectral peak brilliance.

\subsection{Experimental results: control of undulator radiation bandwidth}

The LPA electron beam is experimentally focused on the slit in the chicane center and then refocused in an in-vacuum undulator of $18.16 \mathrm{~mm}$-long period with 107 periods (Figs. 2h-k). While the electrons are dumped at the undulator exit, the undulator radiation is observed using an imaging spectrometer that permits to measure the spatiospectral distribution of the undulator radiation in the far-field (Fig. 6a) and extract the on-axis spectrum (Fig. 6b). As the various contributions to the broadening of the bandwidth are not symmetrically distributed (Eq. (10)), the on-axis distribution is fitted with an asymmetric Gaussian distribution of the form:

$$
\rho(\Lambda)=a \times \exp -\frac{\Lambda^{2}}{2} \times\left[1+\operatorname{erf}\left(\alpha \frac{\Lambda}{\sqrt{2}}\right)\right] \quad \text { with } \quad \Lambda=\frac{\lambda-\lambda_{0}}{\sigma_{\lambda}},
$$

with $\lambda_{0}$ the central value of the radiation, and $\sigma_{\lambda}$ the radiation bandwidth for a pure Gaussian distribution. As the slit is closed, the relative bandwidth decreases (Fig. 6c). The experimental observations are in good agreement with the analytical trend of the undulator radiation bandwidth (Eq. (10)). 

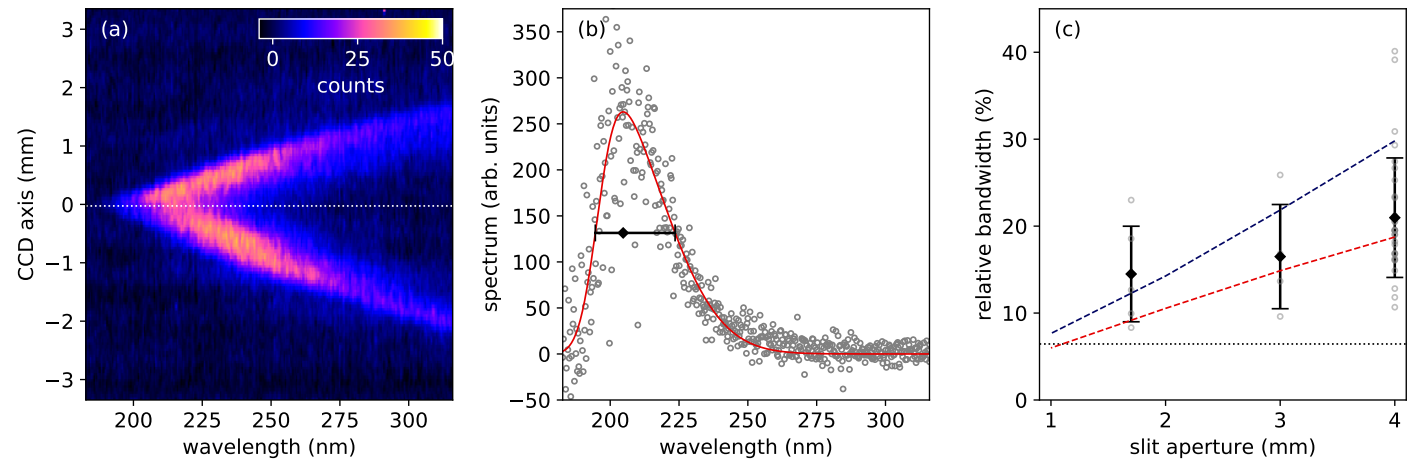

Figure 6. (a) Typical experimental observation of a far-field spatio-spectral distribution of spontaneous undulator radiation for an undulator parameter $K_{U}=1.88$ and an electron slit aperture of $4 \mathrm{~mm}$. (b) Associated on-axis spectrum (horizontal cut along the dashed line in (a)): experimental data (grey dots) and fit (red line). The black diamond indicates the central wavelength and the horizontal error bar corresponds to the full-width at half maximum. (c) Relative bandwidth versus electron slit aperture: single-shot experimental data (grey dots) and average (black), theoretical relative bandwidth for relative energy spread inhomogeneous broadening (red) plus horizontal divergence contribution (blue). The horizontal black line indicates the resolution limit of the spectrometer.

\section{Conclusion}

In this paper, we have reported on a phase-space manipulation of LPA electron beams using a slit inserted in a magnetic chicane. Using numerical simulations, we have shown that it is possible to control the transmitted energy spread along a transfer line taking advantage of the horizontal dispersion in the center of the chicane. The simulations have been performed using measured experimental distributions at the gas jet exit. We thus have shown that despite an almost 100\% initial energy spread, the relevant electrons at the nominal energy are preserved along the beamline while the electrons off-momenta vanish. The proper energy spread tuning enables the control of the undulator radiation bandwidth and therefore an improvement of the expected spectral peak brilliance. Thanks to the manipulation of an LPA beam along the COXINEL line, we have experimentally observed the typical spatio-spectral pattern of the undulator radiation and controlled its on-axis radiation bandwidth [54].

\section{Acknowledgments}

This work was supported by the European Research Council for the Advanced Grants COXINEL (340015, PI: M.-E. Couprie) and X-Five (339128, PI: V. Malka), the LABEX CEMPI (ANR-11-LABX-0007), Ministry of Higher Education and Research, Hauts de France council and European Regional Development Fund (Contrat de Projets EtatRegion CPER Photonics for Society P4S). 
Energy spread tuning of a laser-plasma accelerated electron beam in a magnetic chicane12

\section{References}

[1] Tajima $\mathrm{T}$ and Dawson J $\mathrm{M} 1979$ Phys. Rev. Lett. 43(4) 267-270 URL https://link.aps.org/doi/10.1103/PhysRevLett.43.267

[2] Esarey E, Schroeder C B and Leemans W P 2009 Rev. Mod. Phys. 81(3) 1229-1285 URL https://link.aps.org/doi/10.1103/RevModPhys.81.1229

[3] Wang X, Zgadzaj R, Fazel N, Li Z, Yi S A, Zhang X, Henderson W, Chang Y Y, Korzekwa R, Tsai H E, Pai C H, Quevedo H, Dyer G, Gaul E, Martinez M, Bernstein A C, Borger T, Spinks M, Donovan M, Khudik V, Shvets G, Ditmire T and Downer M C 2013 Nature Communications 41988

[4] Leemans W P, Gonsalves A J, Mao H S, Nakamura K, Benedetti C, Schroeder C B, Tóth C, Daniels J, Mittelberger D E, Bulanov S S, Vay J L, Geddes C G R and Esarey E 2014 Phys. Rev. Lett. 113(24) 245002 URL https://link.aps.org/doi/10.1103/PhysRevLett.113.245002

[5] Gonsalves A J, Nakamura K, Daniels J, Benedetti C, Pieronek C, de Raadt T C H, Steinke S, Bin J H, Bulanov S S, van Tilborg J, Geddes C G R, Schroeder C B, Tóth C, Esarey E, Swanson K, Fan-Chiang L, Bagdasarov G, Bobrova N, Gasilov V, Korn G, Sasorov P and Leemans W P 2019 Phys. Rev. Lett. 122(8) 084801 URL https://link.aps.org/doi/10.1103/PhysRevLett.122.084801

[6] Tsai H E, Swanson K K, Barber S K, Lehe R, Mao H S, Mittelberger D E, Steinke S, Nakamura K, van Tilborg J, Schroeder C, Esarey E, Geddes C G R and Leemans W 2018 Physics of Plasmas 25043107 (Preprint https://doi.org/10.1063/1.5023694) URL https://doi.org/10.1063/1.5023694

[7] Schmid K, Buck A, Sears C M S, Mikhailova J M, Tautz R, Herrmann D, Geissler M, Krausz F and Veisz L 2010 Phys. Rev. ST Accel. Beams 13(9) 091301 URL https://link.aps.org/doi/10.1103/PhysRevSTAB.13.091301

[8] Buck A, Wenz J, Xu J, Khrennikov K, Schmid K, Heigoldt M, Mikhailova J M, Geissler M, Shen B, Krausz F, Karsch S and Veisz L 2013 Phys. Rev. Lett. 110(18) 185006 URL https://link.aps.org/doi/10.1103/PhysRevLett.110.185006

[9] Guillaume E, Döpp A, Thaury C, Ta Phuoc K, Lifschitz A, Grittani G, Goddet J P, Tafzi A, Chou S W, Veisz L and Malka V 2015 Phys. Rev. Lett. 115(15) 155002 URL https://link.aps.org/doi/10.1103/PhysRevLett.115.155002

[10] Thaury C, Guillaume E, Lifschitz A, Ta Phuoc K, Hansson M, Grittani G, Gautier J, Goddet J P, Tafzi A, Lundh O and Malka V 2015 Scientific Reports 516310 EP -

[11] Faure J, Rechatin C, Norlin A, Lifschitz A, Glinec Y and Malka V 2006 Nature 444 737-739

[12] Geddes C G, Rykovanov S, Matlis N H, Steinke S, Vay J L, Esarey E H, Ludewigt B, Nakamura K, Quiter B J, Schroeder C B, Toth C and Leemans W P 2015 Nuclear Instruments and Methods in Physics Research Section B: Beam Interactions with Materials and Atoms 350116 - 121 ISSN 0168-583X URL http://www.sciencedirect.com/science/article/pii/S0168583X15000269

[13] Pak A, Marsh K A, Martins S F, Lu W, Mori W B and Joshi C 2010 Phys. Rev. Lett. 104(2) 025003 URL https://link.aps.org/doi/10.1103/PhysRevLett.104.025003

[14] McGuffey C, Thomas A G R, Schumaker W, Matsuoka T, Chvykov V, Dollar F J, Kalintchenko G, Yanovsky V, Maksimchuk A, Krushelnick K, Bychenkov V Y, Glazyrin I V and Karpeev A V 2010 Phys. Rev. Lett. 104(2) 025004 URL https://link.aps.org/doi/10.1103/PhysRevLett.104.025004

[15] Clayton C E, Ralph J E, Albert F, Fonseca R A, Glenzer S H, Joshi C, Lu W, Marsh K A, Martins S F, Mori W B, Pak A, Tsung F S, Pollock B B, Ross J S, Silva L O and Froula D H 2010 Phys. Rev. Lett. 105(10) 105003 URL https://link.aps.org/doi/10.1103/PhysRevLett.105.105003

[16] Ren C, Duda B J, Hemker R G, Mori W B, Katsouleas T, Antonsen T M and Mora P 2001 Phys. Rev. E 63(2) 026411 URL https://link.aps.org/doi/10.1103/PhysRevE.63.026411 
Energy spread tuning of a laser-plasma accelerated electron beam in a magnetic chicane13

[17] Kalmykov S Y, Yi S A, Beck A, Lifschitz A F, Davoine X, Lefebvre E, Khudik V, Shvets G and Downer M C 2010 Plasma Physics and Controlled Fusion 53014006 URL https : //doi .org/10.1088\%2F0741-3335\%2F53\%2F1\%2F014006

[18] Gonsalves A J, Nakamura K, Lin C, Panasenko D, Shiraishi S, Sokollik T, Benedetti C, Schroeder C B, Geddes C G R, van Tilborg J, Osterhoff J, Esarey E, Toth C and Leemans W P 2011 Nature Physics $\mathbf{7} 862-866$

[19] Palastro J P, Gordon D, Hafizi B, Johnson L A, Peano J, Hubbard R F, Helle M and Kaganovich D 2015 Physics of Plasmas 22123101

[20] Rechatin C, Faure J, Ben-Ismail A, Lim J, Fitour R, Specka A, Videau H, Tafzi A, Burgy F and Malka V 2009 Phys. Rev. Lett. 102(16) 164801 URL https://link.aps.org/doi/10.1103/PhysRevLett.102.164801

[21] Grüner F, Becker S, Schramm U, Eichner T, Fuchs M, Weingartner R, Habs D, Meyer-ter Vehn J, Geissler M, Ferrario M, Serafini L, van der Geer B, Backe H, Lauth W and Reiche S 2007 Applied Physics B 86 431-435 URL https://doi.org/10.1007/s00340-006-2565-7

[22] Couperus J P, Pausch R, Köhler A, Zarini O, Krämer J M, Garten M, Huebl A, Gebhardt R, Helbig U, Bock S, Zeil K, Debus A, Bussmann M, Schramm U and Irman A 2017 Nature Communications 8487

[23] 1984 Optics Communications $50 \quad 373 \quad-\quad 378$ ISSN 0030-4018 URL http://www.sciencedirect.com/science/article/pii/0030401884901056

[24] Kuschel S, Hollatz D, Heinemann T, Karger O, Schwab M B, Ullmann D, Knetsch A, Seidel A, Rödel C, Yeung M, Leier M, Blinne A, Ding H, Kurz T, Corvan D J, Sävert A, Karsch S, Kaluza M C, Hidding B and Zepf M 2016 Phys. Rev. Accel. Beams 19(7) 071301 URL https://link.aps.org/doi/10.1103/PhysRevAccelBeams.19.071301

[25] Thaury C, Guillaume E, Döpp A, Lehe R, Lifschitz A, Ta Phuoc K, Gautier J, Goddet J P, Tafzi A, Flacco A, Tissandier F, Sebban S, Rousse A and Malka V 2015 Nature Communications 6 6860

[26] van Tilborg J, Steinke S, Geddes C G R, Matlis N H, Shaw B H, Gonsalves A J, Huijts J V, Nakamura K, Daniels J, Schroeder C B, Benedetti C, Esarey E, Bulanov S S, Bobrova N A, Sasorov P V and Leemans W P 2015 Phys. Rev. Lett. 115(18) 184802 URL https://link.aps.org/doi/10.1103/PhysRevLett.115.184802

[27] Ferran Pousa A, Martinez de la Ossa A, Brinkmann R and Assmann R W 2019 Phys. Rev. Lett. 123(5) 054801

[28] Eichner T, Grüner F, Becker S, Fuchs M, Habs D, Weingartner R, Schramm U, Backe H, Kunz P and Lauth W 2007 Phys. Rev. ST Accel. Beams 10(8) 082401 URL https://link.aps.org/doi/10.1103/PhysRevSTAB.10.082401

[29] Ghaith A, Kitegi C, André T, Valléau M, Marteau F, Vétéran J, Blache F, Benabderrahmane C, Cosson O, Forest F, Jivkov P, Lancelot J and Couprie M 2018 Nuclear Instruments and Methods in Physics Research Section A: Accelerators, Spectrometers, Detectors and Associated Equipment $909290-293$ ISSN 0168-9002 3rd European Advanced Accelerator Concepts workshop (EAAC2017) URL http://www.sciencedirect.com/science/article/pii/S0168900218302869

[30] Couprie M E, Loulergue A, Labat M, Lehe R and Malka V 2014 Journal of Physics B: Atomic, Molecular and Optical Physics 47234001

[31] Maier A R, Meseck A, Reiche S, Schroeder C B, Seggebrock T and Grüner F 2012 Phys. Rev. X 2(3) 031019 URL https://link.aps.org/doi/10.1103/PhysRevX.2.031019

[32] Smith T I, Madey J M J, Elias L R and Deacon D A G 1979 Journal of Applied Physics 50 $4580-4583$

[33] Huang Z, Ding Y and Schroeder C B 2012 Phys. Rev. Lett. 109(20) 204801 URL https://link.aps.org/doi/10.1103/PhysRevLett.109.204801

[34] Loulergue A, Labat M, Evain C, Benabderrahmane C, Malka V and Couprie M E 2015 New Journal of Physics 17023028 
Energy spread tuning of a laser-plasma accelerated electron beam in a magnetic chicane14

[35] Chen M, Sheng Z M, Ma Y Y and Zhang J 2006 Journal of Applied Physics 99056109 (Preprint https://doi.org/10.1063/1.2179194) URL https://doi.org/10.1063/1.2179194

[36] Glinec Y, Faure J, Guemnie-Tafo A, Malka V, Monard H, Larbre J P, De Waele V, Marignier J L and Mostafavi M 2006 Review of Scientific Instruments 77103301 (Preprint https://doi.org/10.1063/1.2360988) URL https://doi.org/10.1063/1.2360988

[37] Mangles S P D, Thomas A G R, Kaluza M C, Lundh O, Lindau F, Persson A, Tsung F S, Najmudin Z, Mori W B, Wahlström C G and Krushelnick K 2006 Phys. Rev. Lett. 96(21) 215001 URL https://link.aps.org/doi/10.1103/PhysRevLett.96.215001

[38] Couprie M E, Labat M, Evain C, Marteau F, Briquez F, Khojoyan M, Benabderrahmane C, Chapuis L, Hubert N, Bourassin-Bouchet C, Ajjouri M E, Bouvet F, Dietrich Y, Valléau M, Sharma G, Yang W, Marcouillé O, Vétéran J, Berteaud P, Ajjouri T E, Cassinari L, Thaury C, Lambert G, Andriyash I, Malka V, Davoine X, Tordeux M A, Miron C, Zerbib D, Tavakoli K, Marlats J L, Tilmont M, Rommeluère P, Duval J P, N'Guyen M H, Rouqier A, Vanderbergue M, Herbeaux C, Sebdouai M, Lestrade A, Leclercq N, Dennetière D, Thomasset M, Polack F, Bielawski S, Szwaj C and Loulergue A 2016 Plasma Physics and Controlled Fusion 58034020

[39] Couprie M E, Labat M, Evain C, Szwaj C, Bielawski S, Hubert N, Benabderrahmane C, Briquez F, Chapuis L, Marteau F, Valléau M, Marcouillé O, Marchand P, Diop M, Marlats J L, Tavakoli K, Zerbib D, Cassinari L, Bouvet F, Herbeaux C, Bourassin-Bouchet C, Dennetière D, Polack F, Lestrade A, Khojoyan M, Yang W, Sharma G, Morin P and Loulergue A 2016 Journal of Modern Optics 63 309-323

[40] Couprie M E 2018 Nuclear Instruments and Methods in Physics Research Section A: Accelerators, Spectrometers, Detectors and Associated Equipment $909-5-15$ ISSN 0168-9002 3rd European Advanced Accelerator Concepts workshop (EAAC2017) URL http://www.sciencedirect.com/science/article/pii/S0168900218302729

[41] Couprie M E, André T, Blache F, Bouvet F, Briquez F, Dennetire D, Dietrich Y, de Oliviera C, Duval J P, Ajjouri M E, Ghaith A, Herbeaux C, Hubert N, Khojoyan M, Kitégi C, Labat M, Leclercq N, Lestrade A, Loulergue A, Marcouillé O, Marteau F, Oumbarek D, Polack F, Rommelure P, Sebdaoui M, Tavakoli K, Valléau M, Andriyash I A, Benabderrahmane C, Corde S, Gautier J, Goddet J P, Lambert G, Mahieu B, Phuoc K T, Tafzi A, Thaury C, Smartsev S, Malka V, Roussel E, Evain C, Szwaj C and Bielawski S 2019 AIP Conference Proceedings 2054 030034 URL https://aip.scitation.org/doi/abs/10.1063/1.5084597

[42] Marteau F, Ghaith A, N'Gotta P, Benabderrahmane C, Valléau M, Kitegi C, Loulergue A, Vétéran J, Sebdaoui M, André T, Le Bec G, Chavanne J, Vallerand C, Oumbarek D, Cosson O, Forest F, Jivkov P, Lancelot J L and Couprie M E 2017 Applied Physics Letters 111253503 (Preprint https://doi.org/10.1063/1.4986856) URL https://doi.org/10.1063/1.4986856

[43] Ghaith A, Oumbarek D, Kitégi C, Valléau M, Marteau F and Couprie M E 2019 Instruments 3 ISSN 2410-390X URL https://www.mdpi.com/2410-390X/3/2/27

[44] André T, Andriyash I A, Loulergue A, Labat M, Roussel E, Ghaith A, Khojoyan M, Thaury C, Valléau M, Briquez F, Marteau F, Tavakoli K, N'Gotta P, Dietrich Y, Lambert G, Malka V, Benabderrahmane C, Vétéran J, Chapuis L, El Ajjouri T, Sebdaoui M, Hubert N, Marcouillé O, Berteaud P, Leclercq N, El Ajjouri M, Rommeluère P, Bouvet F, Duval J P, Kitegi C, Blache F, Mahieu B, Corde S, Gautier J, Ta Phuoc K, Goddet J P, Lestrade A, Herbeaux C, Évain C, Szwaj C, Bielawski S, Tafzi A, Rousseau P, Smartsev S, Polack F, Dennetière D, Bourassin-Bouchet C, De Oliveira C and Couprie M E 2018 Nature Communications 91334

[45] Scisciò M, Migliorati M, Palumbo L and Antici P 2018 Scientific Reports 86299

[46] Borland M elegant: A Flexible SDDS-Compliant Code for Accelerator Simulation Advanced Photon Source, LS-287, September 2000

[47] Oumbarek Espinos D, Ghaith A, André T, Kitégi C, Sebdaoui M, Loulergue A, Marteau F, Blache F, Valléau M, Labat M, Lestrade A, Roussel E, Thaury C, Corde S, Lambert G, Kononenko O, Goddet J P, Tafzi A, Malka V and Couprie M E 2019 Applied Sciences 9 ISSN 2076-3417 URL https://www.mdpi.com/2076-3417/9/12/2447 
Energy spread tuning of a laser-plasma accelerated electron beam in a magnetic chicane15

[48] Labat M, Loulergue A, Andre T, Andriyash I A, Ghaith A, Khojoyan M, Marteau F, Valléau M, Briquez F, Benabderrahmane C, Marcouillé O, Evain $\mathrm{C}$ and Couprie M E 2018 Phys. Rev. Accel. Beams 21(11) 114802 URL https://link.aps.org/doi/10.1103/PhysRevAccelBeams.21.114802

[49] Espinos D O, Ghaith A, Loulergue A, André T, Kitégi C, Sebdaoui M, Marteau F, Blache F, Valléau M, Labat M, Lestrade A, Roussel E, Thaury C, Corde S, Lambert G, Kononenko O, Goddet J P, Tafzi A, Andriyash I, Malka V and Couprie M E 2020 Plasma Physics and Controlled Fusion 62034001 URL https://doi.org/10.1088\%2F1361-6587\%2Fab5fec

[50] Khojoyan M, Briquez F, Labat M, Loulergue A, Marcouillé O, Marteau F, Sharma G and Couprie M 2016 Nuclear Instruments and Methods in Physics Research Section A: Accelerators, Spectrometers, Detectors and Associated Equipment 829260 - 264 ISSN 0168-9002 2nd European Advanced Accelerator Concepts Workshop - EAAC 2015 URL http://www.sciencedirect.com/science/article/pii/S0168900216001923

[51] Wiedemann H 1999 Particle accelerator physics I (Berlin, Germany: Springer) ISBN 9783540490432, 9783662038277 URL http://www.springer.com/us/book/9783662038277

[52] Kim K J 1986 Nuclear Instruments and Methods in Physics Research Section A: Accelerators, Spectrometers, Detectors and Associated Equipment 24671 - 76 ISSN 0168-9002 URL http://www.sciencedirect.com/science/article/pii/0168900286900483

[53] Walker R P 2019 Phys. Rev. Accel. Beams 22(5) 050704 URL https://link.aps.org/doi/10.1103/PhysRevAccelBeams. 22.050704

[54] Ghaith A, Oumbarek D, Roussel E, Corde S, Labat M, André T, Loulergue A, Andriyash I A, Chubar O, Kononenko O, Smartsev S, Marcouillé O, Kitégi C, Marteau F, Valléau M, Thaury C, Gautier J, Sebban S, Tafzi A, Blache F, Briquez F, Tavakoli K, Carcy A, Bouvet F, Dietrich Y, Lambert G, Hubert N, El Ajjouri M, Polack F, Dennetière D, Leclercq N, Rommeluère P, Duval J P, Sebdaoui M, Bourgoin C, Lestrade A, Benabderrahmane C, Vétéran J, Berteaud P, De Oliveira C, Goddet J P, Herbeaux C, Szwaj C, Bielawski S, Malka V and Couprie M E 2019 Scientific Reports 919020

[55] Ghaith A 2019 Towards compact and advanced free electron laser Ph.D. thesis Université ParisSaclay

[56] Schmüser P, Dohlus M, Rossbach J and Behrens C 2014 Free-Electron Lasers in the Ultraviolet and X-Ray Regime 